\title{
Referentes internacionales para el rediseño de un plan de estudios: competencias para una carrera en Informática
}

Key international references for the redesign of a curriculum: competences for
a Computer Science program

Referências internacionais para o redesenho de um plano de estudos: habilidades para uma carreira em Informática

\section{Gabriela Garita-González ${ }^{1}$ Johnny Villalobos-Murillo ${ }^{1}$ Carmen Cordero-Esquivel $^{1}$ • Sandra Cabrera-Alzate ${ }^{1}$}

Received: Feb/05/2020 • Accepted: Apr/28/2020 • Published: Jan/31/2021

\section{Resumen}

El objetivo de esta investigación fue identificar y contrastar referentes internacionales en busca de estándares de calidad para la definición de los principales componentes de una carrera de formación superior en Computación. La investigación cualitativa y exploratoria inició en enero de 2019, con un equipo de 21 académicos universitarios e investigadores expertos; la técnica focus group se aplicó en tres talleres realizados con personal y autoridades de la carrera de Sistemas de Información de la Universidad Nacional en Costa Rica. Uno de los resultados principales fue la construcción de una base consensuada de competencias profesionales formativas del grado de bachillerato con una base troncal en Computación y el énfasis en Sistemas de Información. Se concluye que los componentes diseñados contemplan los criterios técnicos internacionales y, en especial, el perfil de salida que se puntualizó considera las competencias técnicas, técnicas del énfasis y las transversales.

Palabras clave: innovación curricular; informática y computación; educación superior; referentes universales.

\section{Abstract}

The objective of this research was to identify and contrast key international references in search of quality parameters to define the main components of a higher education computer program. This qualitative and exploratory research began in January 2019 with a team of 21 university academics and expert researchers. The focus group technique was used in the three workshops held with staff and authorities from the Information Systems program from Universidad Nacional in Costa Rica. One of the main results was the creation of a consensual list of formative professional competences for the Bachelor's degree having Computer Sciences as

Gabriela Garita-González, \ gabriela.garita.gonzalez@una.cr, (10 https://orcid.org/0000-0002-7813-2770

Johnny Villalobos-Murillo, \jvillalobos@una.cr, (D) https://orcid.org/0000-0003-1670-8989

Carmen Cordero-Esquivel, $\$ ccordero@una.cr, (1) https://orcid.org/0000-0003-4018-1275

Sandra Cabrera-Alzate, $\$ sandra.cabrera.alzate@una.cr,@ https://orcid.org/0000-0002-0780-2327

1 Escuela de Informática, Universidad Nacional, Heredia, Costa Rica 
the common trunk and Information Systems as the emphasis. It is concluded that the designed components include international technical criteria and that, in particular, the exit profile that was established takes into consideration technical competences, technical competences for the emphasis, and transversal competences.

Keywords: Curriculum innovation; informatics and computer science; higher education; key universal references.

\section{Resumo}

Esta pesquisa teve como objetivo identificar e contrastar referências internacionais na busca de padrões de qualidade para a definição dos principais componentes de um curso de ensino superior em Ciências da Computação. A pesquisa qualitativa e exploratória iniciou em janeiro de 2019, com uma equipe de 21 acadêmicos universitários e pesquisadores especializados; a técnica focus group foi aplicada em três workshops realizados com funcionários e autoridades do curso de Sistemas de Informação da Universidade Nacional, na Costa Rica. Um dos principais resultados foi a construção de uma base consensual de habilidades profissionais formativas do bacharelado com uma espinha dorsal em Computação e com ênfase em Sistemas de Informação. Conclui-se que os componentes desenhados consideram os critérios técnicos internacionais e, especialmente, o perfil de saída enfatizado considera as habilidades técnicas, técnicas de ênfase e as transversais.

Palavras-chave: inovação curricular; informática e computação; ensino superior; referências universais.

\section{INTRODUCCIÓN}

La relevancia del presente estudio radica en la necesidad de desarrollar y de potenciar las competencias profesionales formativas en los planes de estudios que, tradicionalmente, se han diseñado o rediseñado por objetivos y contenidos. Se hace, entonces, evidente el panorama de cambio que enfrenta la sociedad ante las reformas educativas para fortalecer las nuevas habilidades blandas en el campo de computación e informática (Araya y Garita, 2019; Sánchez, Sancho, Botella, García, Aluja, Navarro y Balcazar, 2008). El estudio considera los elementos que deben estar presentes en los diseños curriculares, como el desarrollo económico e industrial y de tecnología del país, y marca un inicio para delimitar cuáles áreas de competencias pueden guiar a los académicos a mejorar el actual plan de estudios, desde el contenido y las estrategias didácticas para el desarrollo de competencias profesionales y laborales
(Sánchez, Rosado, Mellado, Santos-Olmoy Fernández-Medina, 2011).

Dicho esto, se analizó un conjunto de referentes internacionales que conllevan a la generación de competencias del perfil de salida del campo disciplinar. Algunas actividades fueron: contrastar planes de estudios, diseños curriculares y programas de asignaturas a partir de un enfoque de formación profesional por competencias que integra, desde varias dimensiones, el desarrollo integral del estudiantado. Tomando en cuenta la propuesta de Tobón $(2007,2009,2017)$ para el diseño y el rediseño curricular, desde las perspectivas de socioformación y del pensamiento complejo (son una actuación integral, para el análisis y la resolución de problemas reales), a fin de innovar en la orientación y en el proceso de gestión curricular, se toman como base referentes nacionales, regionales e internacionales que aseguren la calidad académica e incorporen mejoras en los procesos de enseñanza y aprendizaje del estudiantado. 
A partir de 2018, la División Académica del Consejo Nacional de Rectores (CONA$\mathrm{RE})$ de Costa Rica, en asociación con el programa HICA Harmonisation and Innovation in Central American Higher Education Curricula: Enhancing and Implementing a Regional Qualifications Framework, la Universidad de Barcelona, el Consejo Superior Universitario Centroamericano (CSUCA) y la Unión Europea, a través del programa Erasmus plus, presentaron el primer Marco de Cualificaciones para la Educación Superior Centroamericana (MCESCA). Solano (2018) señala que el MCESCA es un referente regional cuyo propósito, entre otros, no solo es impulsar la innovación curricular, sino también la reforma en los planes de estudios nuevos o los que necesitan de una restructuración; se enfoca en los resultados de aprendizaje del estudiantado. Por tanto, dicho marco de cualificaciones hace suponer la intencionalidad de concordar los programas de formación en la región, así como brindar transparencia y armonización en las titulaciones universitarias de Centroamérica (MCESCA, 2018).

Se identificaron estudios e investigaciones que hacen reflexionar sobre el uso y la evaluación de competencias. Por ejemplo, Sánchez, Sancho, Botella, García, Aluja, Navarro y Balcazar (2008) aplicaron el rediseño del plan de estudios a partir de competencias profesionales en la Facultad de Informática de Universidad Politécnica de Cataluña, según el Espacio Europeo de Educación Superior (EEES) grado de Ingeniería Informática centradas en el estudiante. Asimismo, la Universidad Abierta de Cataluña, en el 2015, realizó un estudio sobre las competencias transversales que necesita un ingeniero en informática y demanda el mercado laboral (Sastre, 2015).

Siguiendo con el tema, el proyecto Tuning en Europa, en el 2001, las propuestas para la educación superior de González y
Wagenaar (2003) y, en el 2004, para América Latina, son una metodología con reconocimiento internacional "construida por las universidades para las universidades" que replantea las estructuras educativas, en las cuales define la educación basada en competencias genéricas y específicas para la formación de perfiles en los planes de estudio. Lo conforman (Beneitone, Esquetini, González, Marty, Siufi, y Wagenaar, 2007) y lo reafirman (Lino, Alanoca, Salem, Quevedo, Garita, Sepúlveda, Hinojosa, Duarte, Alma, Estrada, Bernal, García, Pow-Sang y González, 2013). Desde la Association for Computing Machinery (ACM) y IEEE Computer Society, vinculados como ACM/IEEE/IEEE Computer Society (2016; 2020), se plantea un modelo de competencias para los planes de estudio, como el de sistemas de información (ACM/ AIS MSIS, 2016). Las reformas de los planes de estudios se definen para el 2001 en China con el marco de Dakar, que incluyó a varios países de África. Para Turquía, en 2004, con el interés político como candidato para la integración a la Unión Europea, se sigue el enfoque educativo por competencias, el cual resalta la importancia de las aptitudes y las competencias, y así generar cambios en las políticas educativas a favor del desarrollo de ambas (Unesco, 2015).

Para América Latina, tomando como ejemplo la educación en México, se ha desarrollado el enfoque basado en competencias como alternativa para formar al individuo de forma integral (Trujillo-Segoviano, 2014); lo acompañan estudios que demuestran la necesidad de los valores y de las actitudes en la formación integral de los profesionales (SEP, 2011). Se destaca que el enfoque por competencias es reciente y que ha tenido carencias en su conceptualización, según Díaz-Barriga (2014), en la falta de claridad en la construcción de los planes de estudio. Ahora, toda reforma necesita del recurso didáctico 
adecuado y de docentes capacitados y formados. En Costa Rica, las universidades se centran en conocimientos disciplinarios que dificultan la movilidad de una universidad a otra (De Faria, 2010).

En resumen, hay una nueva orientación para el diseño y rediseño de los planes de estudio y, como inicio del proceso de actualización del plan de estudios de grado Bachillerato Ingeniería en Sistemas de Información de la Escuela de Informática de la UNA, se hace necesaria la revisión de referentes internacionales y de las propuestas respectivas en el uso de competencias profesionales y de resultados de aprendizaje centrados en el estudiantado. Asimismo, se espera ir dejando atrás el modelo tradicional categorizado por áreas y por disciplinas del conocimiento (objetivos, contenidos y epistemologías), considerados como elementos organizadores del currículo. Se concuerda con Tobón (2017) cuando indica que las universidades deben salir de la sociedad industrial y dar paso a la sociedad de la información; por su parte, Vidal y Araya (2012) proponen sobre la información y cómo debe ser organizada de forma metódica para obtener y generar conocimiento. Por consiguiente, la educación por competencias puede ser la oportunidad para promover la tendencia investigativa (Chanta, 2017).

Se justifica la realización de este estudio para el rediseño del plan de estudios en Ingeniería de Sistemas de Información de la Escuela de Informática de la Universidad Nacional (UNA) por lo significativo del desarrollo de nuevas habilidades y competencias ante la demanda de puestos nacientes, debido a los cambios acelerados en materia de tecnologías y de sistemas de información de los últimos años (ACM/AIS, 2016). Además, los planes de estudio deben actualizarse ante las actuales necesidades del mercado: continuidad del negocio, gestión de datos y contenido, arquitectura empresarial, innovación, emprendimiento y gobernanza; entre tanto, se ha mencionado la importancia de las habilidades blandas como pensamiento crítico, creatividad, colaboración, trabajo en equipo, negociación, comunicación efectiva, resolución de problemas, entre otros. Este marco es clave en la necesidad de la Escuela de Informática de la Universidad Naciona por continuar ofreciendo una carrera de calidad y competitiva, de ahí que el rediseño del plan de estudios Ingeniería de Sistemas de Información forma parte de los compromisos de mejoramiento adquiridos en el proceso de reacreditación, el cual ha sido conferido por cuarta vez y hasta el año 2022.

Luego de la presente introducción, se continúa con el marco teórico que enmarca los conceptos básicos y consideraciones metodológicas, se enumera la primera versión de competencias técnicas, técnicas del énfasis y transversales para el perfil de salida; enseguida, se detallan los principales resultados, así como las reflexiones finales hasta definir las conclusiones.

\section{MARCO TEÓRICO}

De manera puntual, la innovación y la reforma de los planes de estudio deben replantearse para el aprendizaje permanente, mientras que la Organización para la Cooperación y el Desarrollo Económicos (OCDE) (2019) compara y analiza los sistemas de educación superior en educación y competencias. Se destaca la recomendación de combinar competencias sociales y emocionales, tales como el pensamiento crítico, la metacognición, la autoeficacia y la relación con los demás, para mencionar algunas. La Unesco (2014) impulsa estrategias de educación para fortalecer el currículo, la pedagogía y la evaluación de resultados de aprendizaje para el estudiantado, promoviendo sistemas 
de educación con aprendizaje de calidad. Así pues, se hace necesario rediseñar los planes de estudios y los sistemas de evaluación que, mayormente, se centren en la adquisición de habilidades. Ahora bien, se debe intentar cambiar la mentalidad de los actores de los sistemas educativos para centrarse en el aprendizaje (Banco Mundial, 2019). Para crear reflexión en la situación actual de los planes de estudio, caracterizados con sobrecarga y centrados en los contenidos, desde la Comisión Europea se atiente el constante apoyo de proyectos y presupuestos para colaborar con dichas reformas curriculares.

\section{Competencias, habilidades, actitu- des y conceptos afines}

Estos conceptos se integran de manera que permiten, entre sí, complementar su acción en los procesos de aprendizaje y para el contexto del rediseño de planes de estudio. Las competencias, para González y Wagenaar (2003) y De Miguel (2006), son combinaciones de conocimientos, habilidades, capacidades y valores. Cano (2008) señala que coexiste una relación entre el conocimiento conceptual, el procedimental y el actitudinal. Las competencias se han clasificado como genéricas, blandas o básicas; específicas, duras y técnicas; $\mathrm{y}$, por último, como transversales o blandas. Una competencia profesional es el conjunto de conocimientos, habilidades y actitudes que describen los resultados del aprendizaje de una titulación o cualificación de un grado académico; se puede decir, de forma correcta, que puede ser aprendida y puede ser enseñada. Para este contexto, se hace uso de los conceptos competencias técnicas, que se refieren a los conocimientos propios del título; y las competencias transversales, que son generales a todo profesional y, en muchos casos, se pueden clasificar en conceptuales, procedimentales y profesionales (Sánchez et al., 2008).
Ahora bien, un objetivo, una tarea, un contenido, una actitud o una capacidad personal no puede considerarse una competencia (López, 2016), pues esto deriva en confusiones y hace que el término se utilice en distintas realidades en los procesos educativos. Paralelo a esto, vemos lo complementario que son las competencias y los saberes (saber conocer, saber hacer, saber ser). Entre ellos hay reciprocidad, porque ambos integran el conocimiento, la habilidad y la actitud (López, 2016).

En el contexto centroamericano (MCESCA, 2018), se usa el término resultados de aprendizaje, que integran: criterios de desempeño, evidencias del desempeño y competencias. Para este estudio, se utilizó competencia técnica, técnica del énfasis y transversal.

\section{Referentes internacionales en com- putación e informática}

\section{MCESCA: nivel bachillerato}

Tiene como propuesta resultados de aprendizaje o competencias de cinco niveles: saberes disciplinarios y profesionales; aplicación de conocimientos; resolución de problemas e innovación; autonomía con responsabilidad personal, laboral y social; comunicación e interacción profesional, cultural y social.

Entre los principales cambios que conlleva esta innovación curricular se encuentran: la incorporación de los resultados de aprendizaje y la reformulación de la organización del currículo de la educación superior. Sus principales características son estar centrado en el estudiante, medir resultados, ser más comprensibles, permitir la comparación y promover la movilidad académica y profesional.

De este modo, cobran importancia en la definición de los títulos y apoyan el diseño de las asignaturas para el estudiantado. En el contexto nacional, el MCESCA es el marco de cualificaciones centroamericano de la 
educación superior para asegurar la calidad universitaria. Así, los resultados de aprendizaje son declaraciones de lo que se espera que un estudiante conozca, comprenda o sea capaz de hacer al final de un periodo de aprendizaje (Framework for Qualifications of the European Higher Educatios Area, 2018) (Ver Anexo 1).

\section{El Consejo Nacional de Rectores (CONARE)}

Es el referente nacional articulador del sistema de educación superior para el desarrollo. De acuerdo con su misión, cumple un papel significativo en el planteamiento de nuevas propuestas de planes de estudio por parte de las universidades públicas y su proceso de innovación, además de su función como ente catalizador de las necesidades del sector y de la sociedad en general. Mediante las vicerrectorías de docencia de las universidades, se asegura la calidad y la pertinencia de los planes de estudio de nuevas carreras y las actualizaciones que se proponen y que, a través de una revisión cuidadosa, este organismo aprueba. También, el Observatorio Laboral de
Profesiones (OLAP), adscrito al CONARE, incide en el proceso de planteamiento de los planes de estudio, aportando información útil que muestra las necesidades y las competencias que los distintos sectores productivos y sociales están demandando de los profesionales que se incorporan al plano laboral. De esta manera y para esta investigación, los actores internacionales, regionales -indicados en los párrafos anteriores- y el CONARE se han sumado como ejes sustanciales dentro de la indagación, como referentes en el planteamiento y la orientación para la formulación, consistente, de las competencias que darán forma al perfil del profesional de la carrera.

\section{Proyecto Tuning Alfa II para informática}

Es una metodología para diseñar las estructuras y los contenidos a partir de competencias profesionales que incluye: perfil de la titulación, el programa de estudios y la trayectoria de quien aprende. Tiene una propuesta de 13 competencias específicas de la disciplina en informática (Tabla 1).

Tabla 1 Competencias especificas del proyecto Tuning Alfa II para informática

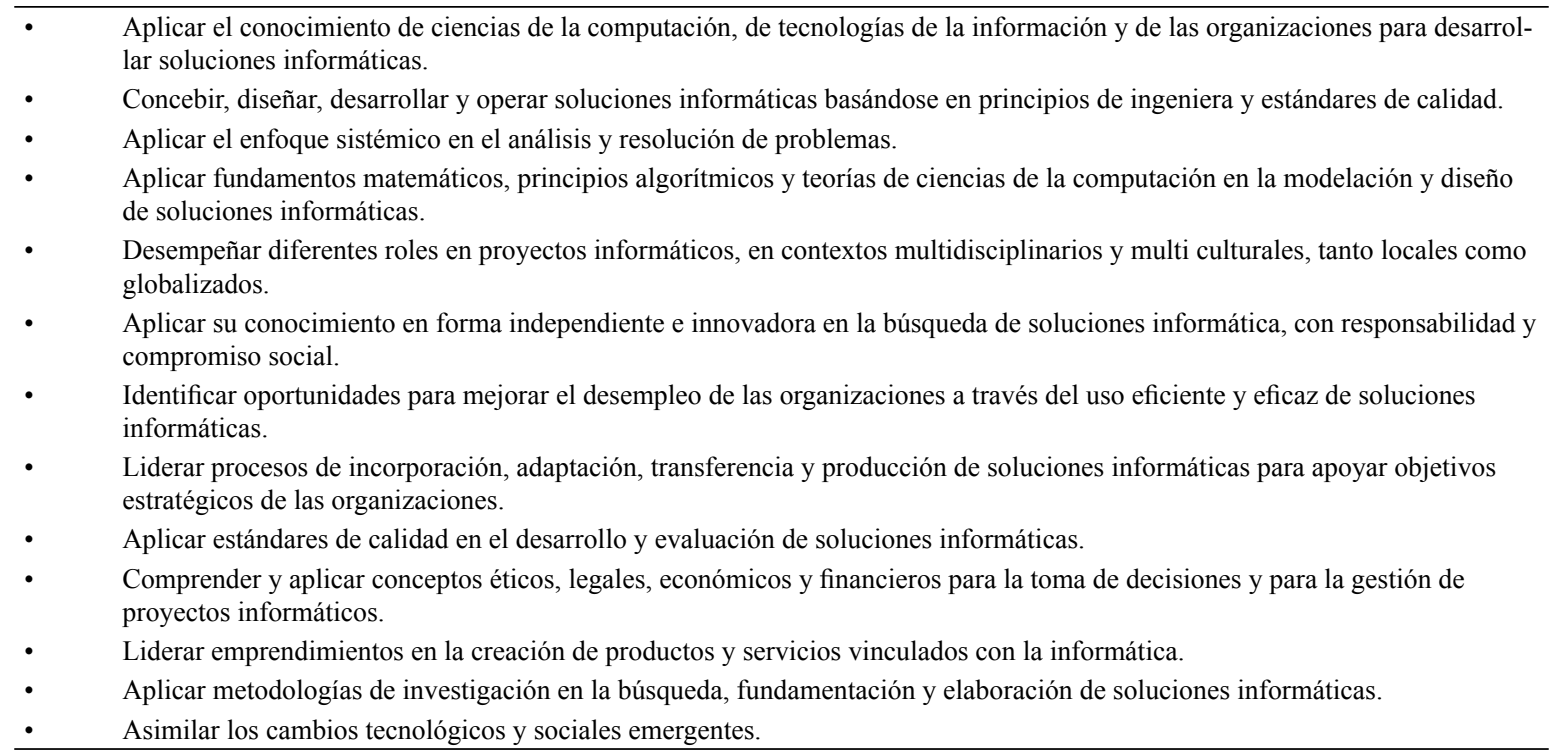

Fuente: Deusto (2015). 
Los diversos escenarios de las carreras en computación e informática apoyan, prácticamente, todos los ámbitos de los sectores de la sociedad, así lo indicaron Vidal y Araña (2012). Siguiendo con el tema, las competencias técnicas para el área informática del proyecto Tuning II (2011 al 2013) definieron el metaperfil de los profesionales graduados, independiente del área de especialización, contextos de formación o del ejercicio (Lino, Alanoca, Salem, Quevedo, Garita, Sepúlveda, Hinojosa, Duarte, Alma Chávez, Estrada, Bernal, García, Pow-Sang y González, 2013). Desde lo general a lo específico, nos lleva a analizar las guías curriculares propuestas por el ACM/AIS/IEEE, las cuales inician por clasificar cinco itinerarios para las carreras Computación e Informática. En adelante, se identifican las competencias generales que aplican a todos los itinerarios: ingeniería informática, ciencias de la computación, sistemas de información, tecnologías de la información e ingeniería de software.

\section{Guías curriculares propuestas por el ACM/IEEE}

El ACM/IEEE, en el 2013, realizó una iniciativa a fin de generar la nomenclatura recomendada por el IEEE para programas universitarios del área computacional en Latinoamérica. Para este estudio se analizó el documento AIS/ACM/IEEE Computing Curricula 2005 (ver Anexo 2), en el cual se actualizaron las competencias profesionales regionales y los cambios del campo. De los principales resultados, se generaron las competencias para ciencias de la computación, sistemas de información, ingeniería de software, ingeniería computacional, tecnologías de la información y otras disciplinas emergentes de híbridos.

La ACM/AIS/IEEE basa su propuesta en cinco itinerarios que clasifican las competencias y dan una visión más específica para el profesional. A la carrera de Computación e Informática de la UNA le interesa revisar el itinerario de sistemas de información, el cual forma parte del perfil actual del plan de estudios. Según esta propuesta curricular para los programas de grado en Sistemas de Información, en que destaca, además, que deben cumplirse los criterios de la acreditación con ABET, se detallan en la Tabla 2 las competencias para ese énfasis.

Tabla 2 Competencias ACM/IEEE para el énfasis SI

\footnotetext{
- Identificar, entender y documentar los requerimientos de sistemas de información.

- Tomar en consideración interfaces hombre máquina y las diferencias interculturales, con el fin de ofrecer una experiencia al usuario de buena calidad.

- Diseñar, implementar, integrar y administrar sistemas de tecnologías de información de arquitectura empresa rial, de datos y de aplicaciones.

- Gestión de proyectos de sistemas de información, incluyendo análisis de riesgos, estudios financieros, presupuestario, contratación y desarrollo y para apreciar los problemas de mantenimiento de sistemas de información.

- Identificar, analizar y comunicar problemas, opciones y alternativas de solución, incluyendo estudios de viabilidad.

- Identificar y comprender las oportunidades creadas por las innovaciones tecnológicas.

- Apreciar las relaciones entre la estrategia de negocio y los sistemas de información arquitectura e infraestructura.
} 
- Comprender los procesos de negocio y la aplicación de tecnología de información para ellos, incluidos los problemas de cambio de gestión, control y riesgo.

- Comprender e implementar arquitecturas, infraestructuras y sistemas seguros.

- $\quad$ Entender los problemas de desempeño y escalabilidad.

- Administrar sistemas de información existentes, incluyendo recursos, mantenimiento, compras y problemas de continuidad del negocio.

Fuente: ACM/IEEE (2013).

\section{Espacio Europeo de Educación Su- perior (EEES)}

El EEES es el órgano regulador que estructura los estudios universitarios en España e identifica competencias que debe tener un titulado con un cierto nivel académico. El Anexo 3 resume las competencias generales propuestas por este órgano para el grado de Ingeniería Informática.

\section{ABET y las competencias para acreditación especializada}

Accreditation Board for Engineering and Technology (ABET) se utiliza en Estados Unidos como referente para definir las competencias profesionales (ver Anexo 4). Asimismo, es el responsable de la acreditación especializada de programas de educación en ciencia aplicada, computación, ingeniería y tecnología.

\section{Conferencia de Decanos y Directo- res de Informática (CODDII)}

En la Conferencia de Decanos y Directores de Informática se ubica el libro blanco sobre las titulaciones de Ingeniería Informática en España en el contexto del EEES (ver anexo 5), que define las competencias básicas del graduando a partir de una ponderación por asignaturas y establece una dedicación del estudiantado por cada una de ellas.

\section{Facultad de Ingeniería de Barcelo- na (FIB)}

Las competencias y el modelo de mapas de competencias de la Universidad Politécnica de Barcelona (FIB) para Ingeniería Informática definió una lista de competencias profesionales (Ver anexo 6). Ahora bien, los referentes internacionales EEES, CODDII, FIB y Tuning II para Latinoamérica están contenidos entre sí y cumplen con el Ministerio de Educación y Ciencia (Gobierno de España, 30 de octubre de 2007, en el Real Decreto 1393/2007). Además, utilizan los descriptores de Dublin (Dublin, 2004) para el grado de Ingeniería Informática, con el fin ordenar los títulos universitarios y determinar las competencias profesionales para el nivel académico correspondiente.

Se detallan las competencias del énfasis de SI, según propuesta curricular del plan de estudio de la FIB (Anexo 7).

Lo dicho hasta aquí sobre los referentes internacionales y su análisis implicó que todos los referentes tienen un contraste de competencias, habilidades y destrezas para computación e informática. Sus semejanzas plantean lógica, ciencias de la computación, fundamentos de matemática, uso procedimental de metodologías de desarrollo, resolución de problemas e integran, a su vez, habilidades blandas como eje transversal que debe tener todo profesional: emprendimiento, comunicación, trabajo en equipo. Las principales diferencias están en el cómo clasifican las competencias.

La primera dificultad del presente estudio es, precisamente, lo que los distingue: el 
estilo de clasificación en las propuestas analizadas. Por lo tanto, mientras que un referente clasifica una competencia como técnica; para otro, es transversal. No obstante, se encontraron similitudes y muchas combinaciones en las propuestas. Entendiendo esto, se determinó que una de las tareas esenciales de la comisión del plan de estudios y de los docentes colaboradores fue la elección de las competencias deseables y la clasificación en el perfil de salida, respecto al entorno de la Escuela de Informática de la UNA donde participaron, en el taller, docentes y empresas del sector TIC. La discusión de estas propuestas para actualizar los planes de estudios contempla, también, variadas propuestas que comparan los referentes internacionales.

El presente estudio se complementa, entonces, con la investigación de Palma y Miñan (2011), en la cual contrastan el Conceive, Desing, Implement and Operative System in the Enterprise and Sociaetal Context (CDIO). Estos investigadores indican que esta codificación se halla contenida en los estándares de acreditación para ingeniería y computación del ABET. A su vez, evidencian que están las definidas por el Proyecto Tuning para Latinoamérica.

En este orden de ideas, se continúa indicando que el International Project Management Asociation (IPMA) contempla elementos para la dirección de proyectos complementarios y necesarios para las ingenierías y se concluye que IPMA contiene CDIO, Tuning y Abet. Se aclara que es una temática especializada en ingenierías. Estos resultados de comparar ABET, EEES, Tuning Informática, ACM/IEEE, FIB y CODDI indican la necesidad de aplicar pilotos holísticos que integren habilidades técnicas y blandas en los planes de estudio de Computación e Informática como estrategia de aprendizaje.

\section{METODOLOGÍA}

La perspectiva del fundamento metodológico fue cualitativa y descriptiva; y el tipo de investigación fue un estudio de caso, donde la herramienta utilizada es la esquematización de experiencias. Esta reflexión conceptual-metodológica se utilizó como base empírica para la comprensión sobre cómo suceden los cambios y su impacto en situaciones o proyectos como el del presente estudio (Tapella y Rodriguez-Bilella, 2014).

\section{Objetivo}

Analizar referentes internacionales para determinar los requisitos necesarios a fin de definir el perfil de salida del egresado del grado de bachillerato en Ingeniería de Sistemas de Información de la Escuela de Informática.

\section{Personas y contexto}

Este estudio inicio en enero del 2018 con la conformación de la comisión del plan de estudios; para el 2019 se finalizó la base de datos de referentes internacionales que fue el instrumento base para la identificación, comparación y reflexión de los grupos docentes. Se contó con una muestra intencional de 21 docentes participantes (10 mujeres). Además, cuatro poseen un doctorado y 17 tienen como grado mayor una maestría. El equipo reúne varias características, pero dos de ellas fueron relevantes para la elección del grupo docente: 1) seis académicos con experiencia en el diseño de planes de estudio y acreditación de carreras de computación e informática en el ámbito nacional y 2) quince son los representantes de cátedras y áreas distintivas de la carrera, a saber: tecnología, matemática, ingeniería de sistemas, sistemas de información e investigación. 


\section{Técnicas}

Durante el desarrollo de esta investigación se destacan dos etapas representativas, la primera fue la aplicación de talleres $\mathrm{y}$, la segunda, la redacción final del conjunto de competencias. La técnica que más se ajustó al contexto de la investigación fue el grupo focal. Se estableció realizar tres talleres en el 2019, en los meses de julio; setiembre; y, por último, en octubre. En cada taller se sensibilizó sobre la problemática, se expuso la propuesta, se revisó la documentación, se analizó cada conjunto de referentes de la base de datos y se reflexionó para aplicar las observaciones y mejoras derivadas de los equipos de docentes. Cabe destacar que esta técnica de grupo focal se aplicó en cada una las reuniones, con el fin de retroalimentar la propuesta (Ver Figura 1).

La segunda etapa fue la redacción final del conjunto de competencias, para cuya técnica de redacción se utilizaron tres documentos fundamentales: 1. La taxonomía de Bloom para informática, 2. La Guía de apoyo para la redacción, puesta en práctica y evaluación de los resultados del aprendizaje, elaborada por La Agencia Nacional de Evaluación de la Calidad y la Acreditación (ANECA, 2013), y 3. La Guía metodológica de diseño y rediseño curricular desde la socioformación y el pensamiento complejo (Tobón, 2017). Se decidió el uso de la fórmula compuesta por verbo de desempeño, contenido conceptual, finalidad y condición de referencia o de contexto (Tabla 3). Cada referente internacional fue redactado con esta técnica de redacción.

\section{RESULTADOS}

Este estudio refiere los procedimientos utilizados en la selección de los referentes internacionales para ser aplicados en los planes de estudios, a fin de que el egresado se convierta en un profesional competente en el mercado laboral. Durante 2019, se inició con la evaluación de 6 propuestas con sus conjuntos de referentes internacionales aplicables a la carrera de Computación e Informática de la Universidad Nacional De Costa Rica, los cuales son reconocidos en el ámbito internacional y que han formado parte de los currículos en Europa y Estados

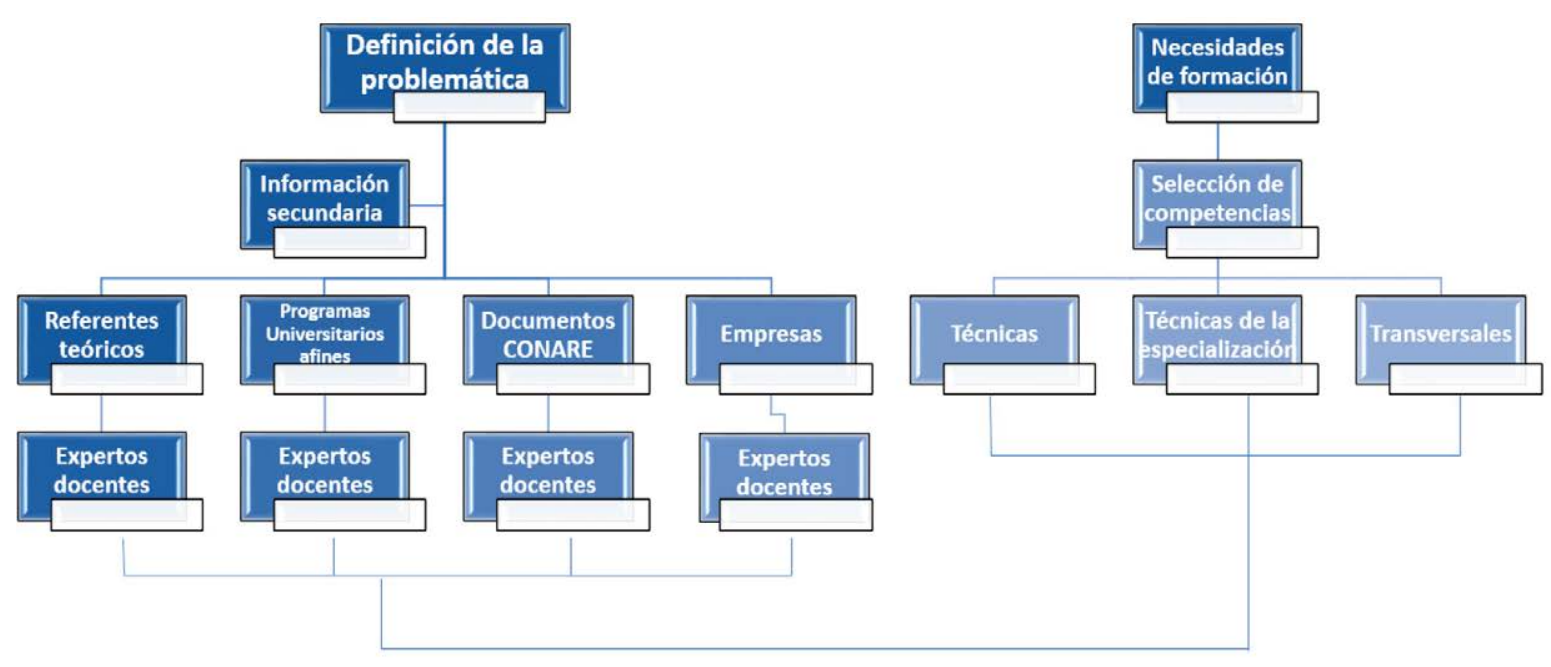

Figura 1. Esquema de metodología utilizada para la experiencia Fuente propia de la investigación. 
Tabla 3 Fórmula para la redacción de una competencia

\begin{tabular}{llll}
\hline \multicolumn{1}{c}{ Verbo de desempeño } & Contenido conceptual & \multicolumn{1}{c}{ Finalidad } & Condición de contexto \\
\hline Es un verbo de acción: & Es el objeto conceptual & Consiste en determinar & Son los referentes, cri- \\
ejecuta, diseña, planea, & central de la competencia & uno o varios fines esen- & terios, normas, parámet- \\
registra, diagnostica, & & $\begin{array}{l}\text { ciales de la competencia, } \\
\text { considerando la sociedad necesidades o retos }\end{array}$ & que necesariamente se \\
implementa, gestiona, & & del conocimiento. & $\begin{array}{l}\text { deben tener en cuenta } \\
\text { como base o condición } \\
\text { evalúa, sistematiza, audita }\end{array}$ \\
& & & de la competencia. \\
\hline
\end{tabular}

Nota: Tobón, S. (2007; 2017).

Unidos. Según la metodología, se analizaron los principales. Un total de 114 referentes analizados: MCESCA bachillerato (19); Proyecto Alfa Tuning II (13); Guías curriculares ACM/IEEE (36); EEES (12); ABET (12); COODDI (11); FIB (12). Lo dicho hasta aquí concluye que se investigaron 114 competencias de 7 referentes internacionales, los cuales brindan propuestas curriculares para las carreras de Computación e Informática.

Este perfil de salida (lista de competencias), que se presenta como resultado principal, evidenció los diferentes estilos de clasificación en las propuestas analizadas. Por lo tanto, para un referente, una competencia se clasifica como técnica; mientras que, para otro, es transversal. No obstante, se encontraron similitudes y muchas combinaciones en las propuestas. Asimismo, se determinó que una de las tareas más importantes de la comisión del plan de estudios y de los docentes colaboradores fue la elección de las competencias deseables y la clasificación en el perfil de salida, respecto al entorno de la Escuela de Informática de la
UNA. En suma, la experiencia analizó varios referentes internacionales en computación e informática que definen y recomiendan el diseño de planes de estudios y utilizan la terminología por competencias. Estos estándares de la industria muestran un camino por seguir en la cambiante disciplina y promueven las reformas curriculares con propuestas estructuradas, como una guía o un modelo referente.

En la Figura 2, se destaca la propuesta del perfil de salida del grado de bachillerato, seguido de una clasificación de 8 competencias técnicas (CT), 5 competencias del énfasis en SI (CTE) y 9 competencias transversales (CT). En la Figura 3, se detalla cada una de las competencias seleccionadas por la clasificación.

\section{Integrar soluciones de Tecnologías de la Información y Comunicación, en función de los procesos empresariales para satisfacer las necesidades de información de las organizaciones, permitiéndoles alcanzar sus objetivos de forma efectiva}

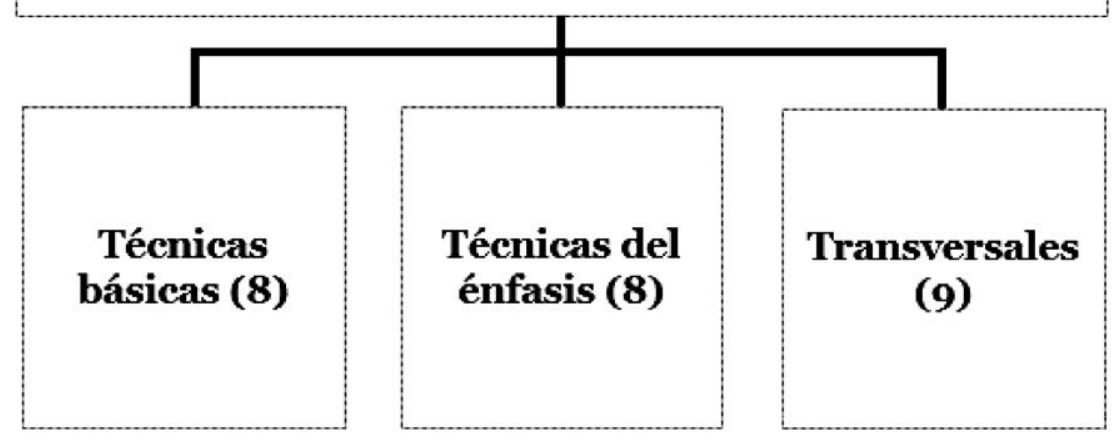

Figura 2. Esquema de competencias formativas (primera versión) Nota: Escuela de Informática. Universidad Nacional. 


\begin{tabular}{|c|c|c|c|}
\hline & Técnicas (CT) & Técnicas del énfasis (CTE) & Transversales (CT) \\
\hline $\begin{array}{l}\text { Integrar soluciones de } \\
\text { tecnologías de la información } \\
\text { y comunicación, en función } \\
\text { de los procesos empresariales } \\
\text { para satisfacer las } \\
\text { necesidades de información } \\
\text { de las organizaciones, } \\
\text { permitiéndoles alcanzar sus } \\
\text { objetivos de forma efectiva }\end{array}$ & 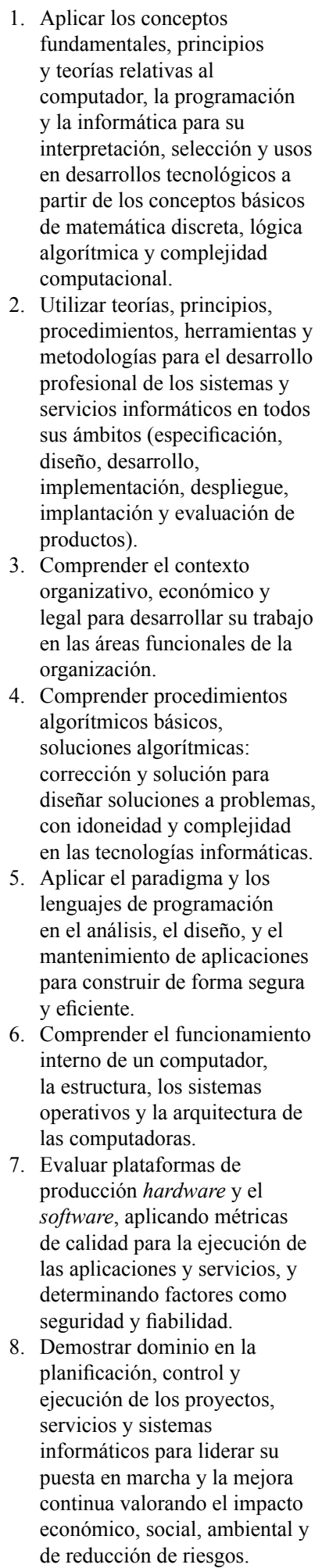 & 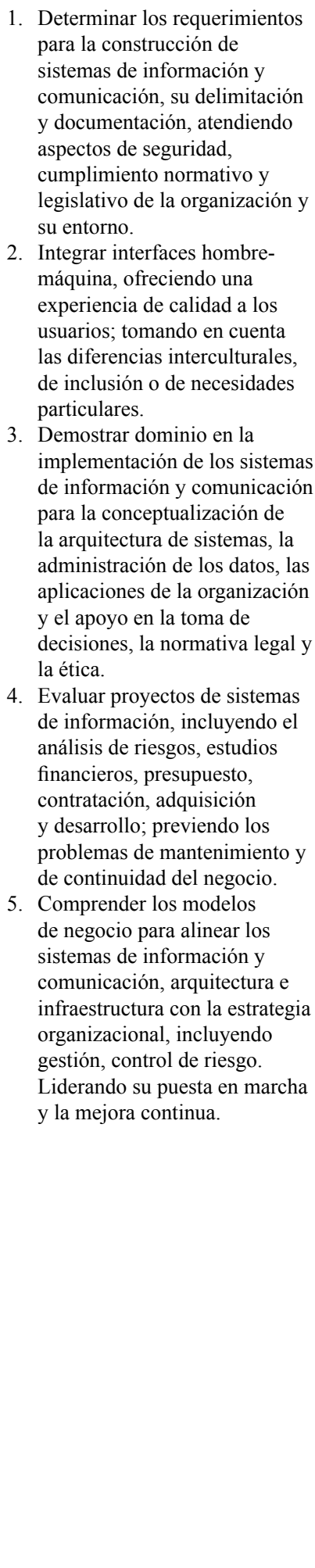 & $\begin{array}{l}\text { 1. Emprendimiento e innovación } \\
\text { 2. Lengua extranjera. } \\
\text { 3. Comunicación eficaz. } \\
\text { 4. Trabajo en equipo. } \\
\text { 5. Aprendizaje autónomo. } \\
\text { 6. Actitud proactiva. } \\
\text { 7. Razonamiento. } \\
\text { 8. Investigación. } \\
\text { 9. Sostenibilidad, cultura y } \\
\text { compromiso social. }\end{array}$ \\
\hline
\end{tabular}

Figura 3. Esquema de competencias formativas (primera versión). Escuela de Informática. Universidad Nacional 


\section{CONCLUSIONES}

La actualización de los planes de estudios es un compromiso permanente con el mejoramiento de la calidad. La tendencia actual impone la transición de los planes de estudios tradicionales, basados en objetivos y contenidos, a un diseño por competencias y resultados de aprendizaje.

El presente estudio se puede dividir en dos ejes principales, el primer eje la identificación y la selección de los referentes universales dio pie a la definición de un conjunto de competencias y el segundo permitió la socialización de hallazgos, mejoras y fortaleció cada una de las actividades académicas.

El perfil de salida que se diseñó con la metodología expuesta considera las competencias técnicas, técnicas del énfasis y las transversales. Este nuevo método deja atrás que la titulación o cualificación sea repartida entre asignaturas, cursos y temática. No hay un criterio definido y quedó a opinión de los expertos de cada área. Hace necesaria más trasversalidad y armonización, para eliminar los vacíos actuales entre las asignaturas, mientras que precisar las competencias previas parece dar solución y apoyar el aumento en los indicadores de aprobación del estudiantado.

Ahora bien, hay otros escenarios que se analizaron: primero, fortalecer el desarrollo de habilidades y destrezas técnicas y blandas; paralelo a esto se está innovando en las reformas educativas del MCESCA como referente regional. Además, se fundamentó en las recomendaciones de referentes internacionales para asegurar la calidad académica y la pertinencia de la disciplina. Se discutieron, dentro del contexto actual, factores económicos, políticos, sociales, geográficos y propios de los sistemas de información del país y el desarrollo a futuro; la necesidad de vinculación con las empresas y el acompañamiento de los principales involucrados; y la intencionalidad. Diversas actividades académicas han surgido a fin de reflexionar ante los cambios necesarios para el rediseño del plan de estudios actual, cuyo propósito se centró en la identificación de referentes para lograr un conjunto de competencias que permitieran definir el perfil de salida del plan de estudios.

Se continúa con el tema de la revisión de los referentes internacionales y locales que fueron la clave para iniciar una base de conocimiento. Es fundamental partir de las necesidades del entorno para diseñar planes de estudios que desarrollen las competencias necesarias en las carreras de Computación e Informática. El rediseño del plan de estudios forma parte de los compromisos de mejoramiento adquiridos en el proceso de acreditación, lo cual ha sido conferido por cuarta vez y hasta el año 2022.

El trabajo articulado y comprometido entre miembros de la comisión, docentes y las instancias universitarias vinculadas al tema de actualización del plan de estudios, así como otras entidades, permitieron el logro de los objetivos previstos para esta primera parte del proceso.

\section{Trabajo futuro}

La experiencia de las ponderaciones que la comisión del plan de estudio realizó fue subjetiva a partir de la experiencia. De forma paralela, se enviaron encuestas de validación con indicadores de importancia para el sector empresarial; y paralelamente, se han iniciado visitas que proyectan 50 empresas de la industria, las cuales tienen o han tenido egresados de la carrera. Esta vinculación con las empresas TIC que dan servicios en el entorno costarricense permitió contrastar la propuesta académica del listado de competencias con los profesionales de la industria. Queda, entonces, la validación con los 
empleadores, generar nuevos resultados y retroalimentar el proceso. Para este 2020, se pretende definir los resultados de aprendizaje para el logro del perfil de salida, correspondiente al conjunto de competencias técnicas, técnicas con énfasis y transversales.

\section{RECONOCIMIENTOS}

Trabajo elaborado en el marco de la actividad académica SIA 0598-18: Evaluación de plan de estudios de la carrera de Ingeniería de Sistemas de Información.

\section{DECLARACIÓN DE LA CONTRIBUCIÓN DE LOS AUTORES}

El porcentaje total de contribución para la conceptualización, preparación y corrección de este artículo fue el siguiente: G.G.G. $30 \%$, J.V.M. $30 \%$ y C.C.E. $20 \%$ S.C.A. $20 \%$.

\section{DECLARACIÓN DE DISPONIBILIDAD DE LOS DATOS}

Los datos que respaldan los resultados de este estudio serán puestos a disposición por el autor correspondiente G.G.G., previa solicitud razonable.

\section{REFERENCIAS}

Agencia Nacional de Evaluación de la Calidad y La Acreditación (ANECA). (2013). Guía de apoyo para la redacción, puesta en práctica y evaluación de los resultados del aprendizaje. Cyan, Proyectos Editoriales. http://www.aneca.es/ Sala-de-prensa/Noticias/2013/ANECA-presenta-la-Guia-para-la-redaccion-y-evaluacion-de-los-resultados-del-aprendizaje.
Araya, E. \& Garita, G. (2019). Propuesta para el fortalecimiento de habilidades técnicas, blandas y complementarias, y su impacto en el currículo TIC desde una perspectiva laboral, profesional y de gestión académica. Revista de Calidad en la Educación Superior, 10(2), 112-141. doi: https://doi.org/10.22458/caes. v10i2.1907.

Association for Computing Machinery (ACM) \& Association form Informations System (AIS) (ACM/AIS) (MSIS) (2016). MSIS 2016 Global Competency Model for Graduate Degree Programs in Information Systems.

Association for Computing Machinery (ACM) \& Association form Informations System (AIS) (ACM/IEEE). (2013). Nomenclatura Recomendada por el IEEE para programas universitarios del área computacional en Latinoamérica. Instituto de Ingenieros Eléctricos y Electrónicos (IEEE).

Association for Computing Machinery (ACM), Insitute of Electrical and Electronics Engineers (IEEE) \& IEEE Computer Society. (2016). Curriculum Guidelines for Undergraduate Degree Programs in Computer Engineering. Instituto de Ingenieros Eléctricos y Electrónicos (IEEE). doi: 10.1145/3025098. https:// www.acm.org/binaries/content/assets/education/ce2016-final-report.pdf

Association for Computing Machinery (ACM), Institute of Electrical and Electronics Engineers (IEEE) \& IEEE Computer Society (IEEECS). (2020). Computer Engineering Curricula 2020. CC2020 Computing Curricula 2020. Paradigmas for Future Computing Curricula (Draft Report). New York: IEEE.

Banco Mundial. (2019). Poner fin a la pobreza de aprendizajes: Una meta para incentivar la alfabetización. Grupo Banco Mundial. https:// www.bancomundial.org/es/news/immersive-story/2019/11/06/a-learning-target-for-alearning-revolution

Beneitone, P.; Esquetini, C.; González, J.; Marty, M.; Siufi, G. \& Wagenaar, R. (eds.). (2007). Tuning América Latina: Reflexiones y perspectivas de la educación superior en América Latina. (Informe Final Proyecto Tuning América Latina 2004-2007). Universidad de Deusto y Universidad de Groningen. http://tuningacademy.org/wp-content/uploads/2014/02/ TuningLAIII_Final-Report_SP.pdf 
Cano, G, M. (2008). La evaluación por competencias en la educación superior. Profesorado. Revista de Curriculum y Formación de Profesorado, 12(3), 1-6. http://www.ub.edu/cubac/ sites/default/files/la evaluacion por competencias_en_la_educacion_superior_0.pdf

Chanta, R. A. (2017). La educación con enfoque por competencias: ¿Una oportunidad para impulsar la investigación en la universidad? Diálogos, 19, 39-49. https://doi.org/10.5377/ dialogos.v0i19.5482

Conferencia de Directores \& Decanos de Ingeniería Informática (CODDII). (2015). Libro blanco del título de grado de la Ingeniería Informática. Coruña, España. http://coddii.org/ libro-blanco-del-titulo-de-grado-de-la-ingieneria-informatica

Consejo Superior Universitario (CSUCA). (2018). Marco de cualificaciones para la educación superior centroamericana (MCESCA). Editorial Serviprensa. http://www.csuca.org/ docs-csuca/libros/Marco\%20de\%20cualificaciones $\% 20$ para $\% 201 \mathrm{l} \% 20$ educacion.pdf

De Faria, E. (2010). La importancia de las competencias en la educación superior. Cuadernos de Investigación y Formación en Educación Matemática, 6, 13-37. https://www.academia. edu/12737848/LO_QUE_LA_INVESTIGACI\%C3\%93N_SABE_ACERCA_DEL USO DE MANIPULATIVOS VIRTUALES_EN_EL_APRENDIZAJE_DE_LA MATEM $\%$ C3\%81TICA

De Miguel, M. (2006). Modalidades de enseñanza centradas en el desarrollo de competencias. Orientaciones para promover el cambio metodológico en el marco del EEES. Universidad de Oviedo. https://www2.ulpgc.es/hege/ almacen/download/42/42376/modalidades ensenanza_competencias_mario_miguel2_ documento.pdf

Deusto. Universidad Deusto. Facultad de Ingeniería (2015). Competencias básicas y generales. Universidad Deusto. Facultad de Ingeniería.

Díaz-Barriga, A. (2014). Construcción de programas de estudio en la perspectiva del enfoque de desarrollo de competencias. Perfiles educativos, 36(143), 142-162. https://doi.org/10.22201/ iisue.24486167e.2014.143.44027

Dublin. (2004). Complete Set Dublin Descriptors 2004 (Reporte inédito). http://iu.foi.hr/index. php/dokumenti/zanimljivi_clanci/complete-set-of-dublin-descriptors468e.pdf
European Higher Education Area (EHEA). (2018). The Framework for Qualifications of the European Higher Education Area (Apéndice III). Conferencia Ministerial del Espacio Europeo de Educación Superior (EEES) París 2018.

Facultad de Informática de Barcelona (FIB). Universidad Politécnica de Catalunya (s. f.). Competencias. Universidad Politécnica de Catalunya. https://www.fib.upc.edu/es/estudios/grados/grado-en-ingenieria-informatica/ plan-de-estudios/competencias

González, J., \& Wagenaar, R. (eds.) (2003). Tuning Educational Structures in Europe. (Informe final. Fase 1). Universidad de Deusto.

Lino, J., Alanoca, J., Salem, J., Quevedo, J., Garita, G., Sepúlveda, R., Hinojosa, C. M., Duarte, H. J., Alma, A. P., Estrada, A. E., Bernal, D., García, M. E., Pow-Sang, A., \& González, L. (2013). Educación superior en América Latina: Reflexiones y perspectivas en informáti$c a$. Universidad de Deusto.

López, E. (2016). En torno al concepto de competencia: Un análisis de fuentes. Revista de Currículum y Formación del Profesorado, 20(1), 311-322. https://recyt.fecyt.es/index.php/ profesorado/article/view/49881/30506

Ministerio de Educación y Ciencia. Gobierno de España. (30 de octubre de 2007). Real Decreto 1393/2007, de 29 de octubre, por el que se establece la ordenación de las enseñanzas universitarias oficiales. DO: BOE, 260, 44037-44048. https://www.boe.es/boe/ dias/2007/10/30/pdfs/A44037-44048.pdf

Organización para la Cooperación y el Desarrollo Económicos (OCDE). (2019). El trabajo de la OCDE sobre educación y competencias. OCDE

Palma, M., Miñán, E., \& de los Ríos, I. (2011). Competencias genéricas en ingeniería: Un estudio comparado en el contexto internacional. In Aeipro (Asociación Española de Dirección e Ingeniería de Proyectos). XV Congreso Internacional de Ingeniería de Proyectos (pp. 2552-2569) Huesca, España: Aeipro. doi: http://dspace.aeipro.com/xmlui/bitstream/ handle/123456789/1553/CIIP11_2552_2569. pdf? sequence $=1 \&$ is Allowed $=\mathrm{y}$

Sánchez, F., Sancho, M., Botella, P., García, J., Aluja, T., Navarro, J., \& Balcazar, J. L. (2008). Competencias profesionales del grado de Ingeniería Informática. In De Carvalho; Llamas, M. \& Silvera, R. (eds.), XIV Jornadas de Enseñanza Universitaria de la Informática 
(JENUI2008) (pp. 147-154). New York, USA: IEE, Sociedade de Educación. doi https:// www.academia.edu/31166408/Competencias_profesionales_para_el_Grado_en_Ingenier $\% \mathrm{C} 3 \% \mathrm{ADa}$ Inform $\% \mathrm{C} 3 \%$ A 1 tica

Sánchez, L. E., Rosado, D. G., Mellado, D., Santos-Olmo, A. y Fernández-Medina, E. (2011). Proceso de reificación de las competencias generales \& específicas para el grado en Ingeniería Informática y Definición de un Plan de Métricas de Evaluación de dichas Competencias. In XVII Jornadas de Enseñanza Universitaria de la Informática (JENUI11) (pp. 51-58). Sevilla, España. https://www. researchgate.net/publication/232252330 Proceso_de_Reificacion_de_las_Competencias_Generales_y_Especificas_para_el_Grado_en_IngenierIa_Informatica_y_Definicion_de_un_Plan_de_Metricas_de_Evaluacion_de_dichas_Competencias

Sánchez, S. J., Salavarrienta, J., Ramírez, V., Jarami1lo, I., \& Durán, J. (2017). ¿Cómo aportan las competencias ABET al currículo operativo del curso de Introducción a la Ingeniería de Sistemas y Computación de la Universidad de Los Andes? https:/www.researchgate.net/ publication/327023623_Como_aportan_las competencias_ABET_al_curriculo_operativo_del_curso_de_Introduccion_a_la_Ingenieria_de_Sistemas_y_Computacion_de_la Universidad de los_Andes

Sastre, J. A. (2015). Competencias profesionales del ingeniero informático. (Ebook Creative Commons). Universidad Abierta de Cataluña. España.

Sistema de Estudios de Posgrado (SEP). (2011). Plan de Estudios 2011, Educación Básica. México.

Solano, V. (2018). Presentación del primer Marco de Cualificaciones para la Educación Superior Centroamericana (MESCA). https://www.conare.ac.cr/noticias/101-presentacion-del-primer-marco-de-cualificaciones-para-la-educacion-superior-centroamericana-mcesca
Tapella, E., \& Rodríguez-Bilella, P. (2014). Sistematización de experiencias: Una metodología para evaluar intervenciones de desarrollo. Revista de Evaluación de Programas y Políticas públicos, 3, 80-116. https://doi.org/10.5944/ reppp.3.2014.13361

Tobón, S. (2007). Formación basada en competencias. Pensamiento complejo, diseño curricular y didáctica. ECOE.

Tobón, S. (2009). Diseño sistémico de módulos en la educación superior. Cife.

Tobón, S. (2017). Guía metodológica de diseño y rediseño curricular desde la socioformación y el pensamiento complejo. Kresearch corp.

Trujillo-Segoviano, J. (2014). El enfoque en competencias y la mejora de la educación. $R a$ Ximhai, 10(5). https://doi.org/10.35197/ rx.10.03.e1.2014.26.jt

United Nations Educational Scientific and Cultural Organization (Organización de las Naciones Unidas para la Educación, la Ciencia y la Cultura (Unesco). (2015). Informe de seguimiento de la EPT en el mundo. La educación para todos, 2000-2015: Logros y desafios.Autor.

United Nations Educational Scientific and Cultural Organization (Organización de las Naciones Unidas para la Educación, la Ciencia y la Cultura (Unesco). (2014). Estrategia de Educación de la UNESCO (2014-2021). Autor.

Universidad Valladolid. (2 de julio de 2010). Real Decreto $861 / 2010$, que modifica el Real Decreto 2393/2007, de 29 de octubre, por el que se establece la ordenación de las enseñanzas universitarias. DO: MemoriaFormalizaVerificaUVa04042011_V3.doc. https://www.inf. uva.es/wp-content/uploads/2014/05/UVaGradoIngenieriaInformatica.pdf

Vidal, M. J., \& Araña, A. B. (2012). Gestión de la información y el conocimiento. Revista $\mathrm{Cu}$ bana de Educación Médica Superior, 26(3), 474-484. http://scielo.sld.cu/pdf/ems/v26n3/ ems13312.pdf

\section{(c) $(1) \Theta \Theta$}

Referentes internacionales para el rediseño de un plan de estudios: competencias para una carrera en Informática (Gabriela Garita-González • Johnny Villalobos-Murillo • Carmen Cordero-Esquivel - Sandra Cabrera-Alzate). Uniciencia is protected by AttributionNonCommercial-NoDerivs 3.0 Unported (CC BY-NC-ND 3.0) 


\section{Anexo 1. MCESCA: resultados de aprendizaje bachillerato (MCESCA, 2018)}

- Demuestra la comprensión y explica los principios y conceptos que sustentan los procedimientos, procesos, sistemas y metodologías de la profesión.

- Demuestra conocimientos de cultura humanística y social que le brindan una visión amplia de su profesión y del entorno.

- Muestra conocimiento sobre el riesgo de desastres en el campo de su especialidad y propone soluciones para reducirlo.

- Domina las tecnologías y herramientas propias de su profesión para la innovación de procedimientos, procesos, sistemas y metodologías para la mejora de resultados de su área.

- Demuestra pensamiento crítico, actitud de indagación y rigor analítico en el planteamiento y la resolución de problemas.

- Aplica conocimiento teórico-práctico, en diferentes contextos para identificar y resolver problemas de su ámbito laboral y colabora en la resolución de problemas complejos.

- Propone e implementa nuevos procedimientos aplicables a la solución de problemas y mejora de su campo profesional.

- Toma decisiones profesionales con base en datos en información pertinente, válida y confiable.

- Demuestra destreza y habilidad en el uso de herramientas metodológicas, tecnológicas, equipo especializado y en la lectura de datos para el ejercicio de su profesión en diversos contextos.

- Demuestra responsabilidad en la determinación de resultados personales y laborales de acuerdo a su función tomando como referencias las normativas legales y éticas de su campo profesional.

- Identifica sus necesidades de actualización, capacitación y formación, durante su proceso formativo y en el ejercicio profesional, y busca los medios para cubrirlas por medios formales e informales, nacionales e internacionales, presenciales y en línea.

- Evalúa su desempeño profesional con base en referentes de calidad, nacionales e internacionales, y la incidencia de sus decisiones en aspectos económicos, sociales, ambientales y de reducción de riesgos.

- Emprende proyectos profesionales para la generación de negocios y para el beneficio social con criterios de pertinencia, calidad, innovación y sustentabilidad.

- Comunica, en varios lenguajes y formatos, y de forma pertinente, propositiva y clara, información de su campo profesional a los diferentes actores, equipos de trabajo y destinatarios de las acciones profesionales.

- Se comunica correctamente en su lengua oficial y utiliza una lengua extranjera con el dominio requerido para el ejercicio de su profesión.

- Utiliza tecnologías digitales para el manejo e interpretación de datos e información de forma apropiada a su nivel y su profesión. Interacción profesional, cultural y social. Demuestra habilidades colaborativas y cooperativas en el campo profesional, cultural y social.

- Lidera y colabora proactivamente en equipos de trabajo y en comunidades profesionales para el logro de objetivos y mejoramiento de la calidad de vida.

- Muestra respeto hacia la diversidad en todas sus manifestaciones y contribuye al bien común.

- Participa en redes de colaboración que fortalezcan su campo profesional. 
Anexo 2. Competencias generales ACM/IEEE (ACM/IEEE, 2013)

- Capacidad intelectual para aplicar los fundamentos matemáticos y teoría de la ciencia computacional.

- Capacidad de tener una perspectiva crítica y creativa en la identificación y solución de problemas utilizando el pensamiento computacional.

- Capacidad intelectual para el reconocimiento del papel central de algoritmos y estructuras de datos.

- Conocer el hardware desde una perspectiva de software, por ejemplo, uso del procesador, memoria, unidades de disco, pantalla, etc.

- Capacidad para implementar algoritmos y estructuras de datos en el software.

- Capacidad de diseñar e implementar unidades estructurales más grandes que utilizan algoritmos y estructuras de datos y las interfaces a través de las cuales se comunican estas unidades.

- Capacidad de aplicar los principios de la ingeniería de software y tecnologías para garantizar que las implementaciones de software son robustas, confiables y adecuadas para el usuario para quien fue diseñado.

- Comprender lo que pueden y no pueden lograr las tecnologías actuales.

- Comprender las limitaciones de computación, incluyendo la diferencia entre lo que la computación es inherentemente incapaz de hacer versus lo que puede lograrse a través de la tecnología y la ciencia futura.

- Comprender los efectos sobre los individuos, organizaciones y la sociedad de la implementación de las intervenciones y soluciones tecnológicas.

- Comprender el concepto del ciclo de vida, incluyendo la importancia de sus fases (planificación, desarrollo, implementación y evolución).

- Entender las implicaciones del ciclo de vida para el desarrollo de todos los aspectos de los sistemas informáticos (incluyendo software, hardware e interfaz persona computadora).

- Entender la relación entre la gestión de la calidad y del ciclo de vida.

- Comprender el concepto esencial del proceso en lo que se refiere a la informática especialmente en la ejecución del programa y funcionamiento del sistema.

- Comprender el concepto esencial del proceso en lo que se refiere a la actividad profesional, especialmente en la relación entre la calidad del producto y el despliegue de los procesos humanos adecuados durante el desarrollo del producto.

- Capacidad de identificar temas de computación avanzada y entender las fronteras de la disciplina.

- Capacidad de expresarse en forma oral y escrita, como se espera de un graduado universitario.

- Capacidad de participar activa y coordinadamente en un equipo.

- Capacidad de identificar eficazmente los objetivos y prioridades de su trabajo / área / proyecto, indicando la acción, el tiempo y los recursos necesarios.

- Capacidad para conectar teoría y habilidades aprendidas en la academia a hechos reales explicando su pertinencia y utilidad.

- Comprender asuntos profesionales, legales, de seguridad, políticos, humanistas, ambientales, culturales y éticos.

- Capacidad de demostrar las actitudes y prioridades que honran, protegen y mejoran la estatura ética de la profesión.

- Capacidad de emprender, completar y presentar un proyecto integrador.

- Comprender la necesidad de aprendizaje durante toda la vida y la mejora de habilidades y destrezas.

- Habilidad para comunicarse en un segundo idioma. 
Anexo 3. Competencias generales EEES (Universidad Valladolid, 2010)

- Capacidad para diseñar, desarrollar, evaluar y asegurar la accesibilidad, ergonomía, usabilidad y seguridad de los sistemas, servicios y aplicaciones informáticas, así como de la información que gestionan.

- Capacidad para definir, evaluar y seleccionar plataformas hardware y software para el desarrollo y la ejecución de sistemas, servicios y aplicaciones informáticas, de acuerdo con los conocimientos adquiridos según lo establecido en las competencias de formación especificadas a continuación en esta sección de la memoria.

- Capacidad para concebir, desarrollar y mantener sistemas, servicios y aplicaciones informáticas empleando los métodos de la ingeniería de software como instrumento para el aseguramiento de su calidad, de acuerdo con los conocimientos adquiridos según lo establecido en las competencias de formación especificadas a continuación en esta sección de la memoria.

- Capacidad para concebir y desarrollar sistemas o arquitecturas informáticas centralizadas o distribuidas integrando hardware, software y redes de acuerdo con los conocimientos adquiridos según lo establecido en las competencias de formación especificadas a continuación en esta sección de la memoria.

- Capacidad para conocer, comprender y aplicar la legislación necesaria durante el desarrollo de la profesión de Ingeniero Técnico en Informática y manejar especificaciones, reglamentos y normas de obligado cumplimiento.

- Conocimiento de las materias básicas y tecnologías, que capaciten para el aprendizaje y desarrollo de nuevos métodos y tecnologías, así como las que les doten de una gran versatilidad para adaptarse a nuevas situaciones.

- Capacidad para resolver problemas con iniciativa, toma de decisiones, autonomía y creatividad.

- Capacidad para saber comunicar y transmitir los conocimientos, habilidades y destrezas de la profesión de Ingeniero Técnico en Informática.

- Conocimientos para la realización de mediciones, cálculos, valoraciones, tasaciones, peritaciones, estudios, informes, planificación de tareas y otros trabajos análogos de informática, de acuerdo con los conocimientos adquiridos según lo establecido en las competencias de formación especificadas a continuación en esta sección de la memoria.

- Capacidad para analizar y valorar el impacto social y medioambiental de las soluciones técnicas, comprendiendo la responsabilidad ética y profesional de la actividad del Ingeniero Técnico en Informática.

- Conocimiento y aplicación de elementos básicos de economía y de gestión de recursos humanos, organización y planificación de proyectos, así como la legislación, regulación y normalización en el ámbito de los proyectos informáticos, de acuerdo con los conocimientos adquiridos según lo establecido en las competencias de formación especificadas a continuación en esta sección de la memoria.

Anexo 4. Competencias ABET (Sánchez, Salavarrienta, Ramírez, Jaramillo y Durán 2017)

- Habilidades para aplicar el conocimiento de matemáticas, ciencia e ingeniería.

- Habilidades para diseñar y conducir experimentos, así como para analizar e interpretar datos.

- Habilidades para diseñar un sistema, componente o proceso que satisfaga las necesidades deseadas teniendo en cuenta restricciones realistas tales como las económicas, ambientales, sociales, políticas, éticas, de salud, y seguridad, manufactura y sostenibilidad.

- Habilidades para desempeñarse en equipos de trabajo multidisciplinarios.

- Habilidades para identificar, formular y resolver problemas de ingeniería. 
- Comprensión de la responsabilidad ética y profesional.

- Habilidades para comunicarse efectivamente.

- Una formación necesaria para entender el impacto de las soluciones de ingeniería en un contexto global y social

- La habilidad y el reconocimiento de la necesidad de aprender a lo largo de la vida.

- Conocimiento de los temas de interés contemporáneos.

- Habilidades para utilizar las técnicas, destrezas y herramientas modernas de la ingeniería necesaria para la práctica de la profesión.

\section{Anexo 5. Competencias CODDII (CODDII, 2015)}

- Aprender de manera autónoma nuevos conocimientos y técnicas adecuadas para la concepción, el desarrollo o la explotación de sistemas informáticos.

- Trabajar en un entorno multidisciplinar y multilingüe.

- Comunicar de forma efectiva, tanto por escrito como oralmente, conocimientos, procedimientos, resultados e ideas relacionadas con las tecnologías de la información y la comunicación (TIC) y, concretamente, de la informática, conociendo su impacto socioeconómico.

- Asumir la responsabilidad social, ética, profesional y civil de la actividad del ingeniero(a) en Informática, así como su papel en el ámbito de las TIC y de la sociedad de la información y del conocimiento.

- Concebir, valorar, planificar y dirigir proyectos informáticos utilizando los principios y metodologías propios de la ingeniería, de la gestión de recursos humanos y de la economía.

- Diseñar, desarrollar, evaluar y asegurar la accesibilidad, ergonomía, usabilidad y seguridad de los sistemas, aplicaciones y servicios informativos, así como de la información que proporcionan, conforme a la legislación y normativa vigentes.

- Definir, evaluar y seleccionar plataformas hardware y software para el desarrollo y la ejecución de aplicaciones y servicios informáticos de diversa complejidad.

- Interpretar, seleccionar, valorar, y crear nuevos conceptos, teorías, usos y desarrollos tecnológicos relacionados con la informática y su aplicación, usando los fundamentos matemáticos, físicos, económicos y sociológicos necesarios.

- Concebir, desarrollar y mantener sistemas, servicios y aplicaciones software con los niveles de calidad exigidos, empleando los métodos de la ingeniería del software y los lenguajes de programación más adecuados.

- Concebir, desarrollar, instalar y mantener sistemas o arquitecturas informáticas, centralizadas o distribuidas, integrando hardware, software y redes.

- Proponer, analizar, validar e interpretar soluciones informáticas para una organización, en situaciones reales y en diversas áreas de aplicación.

- Concebir, desplegar, organizar y gestionar sistemas y servicios informáticos, en contextos empresariales o institucionales, para mejorar sus procesos de negocio, responsabilizándose y liderando su puesta en marcha y mejora continua, así como valorar su impacto económico y social. 


\section{Anexo 6. Competencias técnicas (FIB, s. f.)}

- Demostrar conocimiento y comprensión de hechos esenciales, conceptos, principios y teorías relativas a la informática y a sus disciplinas de referencia.

- Utilizar, de forma apropiada, teorías, procedimientos y herramientas en el desarrollo profesional de la ingeniería informática en todos sus ámbitos (especificación, diseño, implementación, despliegue, implantación y evaluación de productos) de manera que se demuestre la comprensión de los compromisos adoptados en las decisiones de diseño.

- Demostrar conocimiento y comprensión del contexto organizativo, económico y legal en el que desarrolla su trabajo (conocimiento adecuado del concepto de empresa, marco institucional y jurídico de la empresa, organización y gestión de empresas).

- Demostrar conocimiento y capacidad de aplicación de los procedimientos algorítmicos básicos de las tecnologías informáticas para diseñar soluciones a problemas, analizando la idoneidad y la complejidad de los algoritmos.

- Analizar, diseñar, construir y mantener aplicaciones de forma robusta, segura y eficiente, escogiendo el paradigma y los lenguajes de programación más adecuados.

- Demostrar conocimiento y comprensión del funcionamiento interno de un computador y del funcionamiento de las comunicaciones entre ordenadores.

- Evaluar y seleccionar plataformas de producción hardware y software para la ejecución de aplicaciones y de servicios informáticos.

- Planificar, concebir, desplegar y dirigir proyectos, servicios y sistemas informáticos en todos los ámbitos, liderando su puesta en marcha, su mejora continua y valorando su impacto económico y social

\section{Anexo 7. Competencias técnicas para SI (FIB, s. f.)}

- Integrar soluciones de tecnologías de la información y las comunicaciones y procesos empresariales para satisfacer las necesidades de información de las organizaciones, permitiéndoles llegar a sus objetivos de forma efectiva.

- Determinar los requisitos de los sistemas de información y comunicación de una organización, atendiendo aspectos de seguridad y cumplimiento de la normativa y de la legislación vigente.

- Participar activamente en la especificación, el diseño, la implementación y el mantenimiento de los sistemas de información y de comunicación.

- Demostrar comprensión y aplicar los principios y las prácticas de las organizaciones, de manera que puedan ejercerse como enlace entre las comunidades técnica y de gestión de una organización, y participar activamente en la formación de los usuarios. 